\title{
INTERPOLATION AND EXTRAPOLATION OF ROOM TRANSFER FUNCTIONS BASED ON COMMON ACOUSTICAL POLES AND THEIR RESIDUES
}

\author{
Yoichi Haneda and Yutaka Kaneda \\ NTT Human Interface Laboratories \\ 3-9-11, Midori-cho, Musashino-shi, \\ Tokyo, 180, Japan
}

\begin{abstract}
We propose a new method of modeling a room transfer function (RTF) that uses common acoustical poles and their residues. The common acoustical poles correspond to the resonance frequencies (eigenvalues) of the room, and their residues are composed of the eigenfunctions of the source and receiver positions in the room. Because the common acoustical poles do not depend on the source and receiver positions, this model expresses the RTF variations due to changes in the source and receiver positions by using residue variations. We also propose methods of interpolating and extrapolating RTFs based on the proposed commonacoustical-pole and residue model. Computer simulation demonstrated that unknown RTFs can be well estimated from known (measured) RTFs by using these methods.
\end{abstract}

\section{INTRODUCTION}

The room transfer function (RTF), which describes the sound transmission characteristics between a source and a receiver in a room, plays a very important role in acoustic signal processing and sound field control. Because the RTF strongly depends on the source and receiver positions, it should be measured for every source-receiver configuration. However, this is cumbersome. An interpolation or extrapolation technique that could be used to estimate an unknown RTF at an arbitrary position using known RTFs would thus be very attractive.

In our common-acoustical-pole and zero model of RTFs [1], the common acoustical poles correspond to the resonance frequencies of the room. They are estimated as values common to RTFs for different source and receiver positions. Because the common acoustical poles do not depend on the source and receiver positions, the RTF variations due to changes in the source and receiver positions are expressed as variations in the zeros. Fewer parameters are thus needed to express the RTF variations than with the conventional allzero model or the pole/zero model (where poles are estimated as different values for each RTF). However, finding the explicit relationship between the variations in the zeros and the change in the source and receiver positions is difficult.

In this paper, we propose a new model of RTFs that uses the common acoustical poles and their residues to efficiently express the RTF variations due to changes in source and receiver positions. Because the common acoustical poles are fixed values for all RTFs, only the variations in the resicues are used for expressing the RTF variations. The residues are composed of the eigenfunctions of the source and receiver positions in the physical expression of the RTF [2].

We also propose methods for interpolating and extrapolating the RTF at an arbitrary position by using the common acoustical poles and their residues. Because the residue variations due to changes in the source and receiver positions have a regularity that follows the eigenfunctions of the room, we can estimate the residue variations as explicit functions of the positions from known RTFs.

\section{COMMON-ACOUSTICAL-POLE AND RESIDUE MODEL}

The room transfer function $H\left(\omega, \mathbf{r}_{\mathrm{s}}, \mathbf{r}_{0}\right)$ between the source and receiver positions is expressed using resonance frequencies $\omega_{i}$ (eigenvalues) and their eigenfunctions $P_{i}(\mathbf{r})$ [2]:

$$
H\left(\omega, \mathbf{r}_{\mathrm{s}}, \mathbf{r}_{\mathrm{o}}\right)=C_{1} \sum_{i=1}^{\infty} \frac{P_{i}\left(\mathbf{r}_{\mathrm{s}}\right) P_{i}\left(\mathbf{r}_{\mathrm{o}}\right) j \omega}{\left(\omega^{2}-\omega_{i}^{2}-2 j \delta_{i} \omega_{i}\right)},
$$

where $\omega$ is a frequency, $\delta_{i}$ is a damping constant, and $C_{1}$ is a gain constant. The $r_{s}$ and $r_{0}$ represent the position vectors of the source and receiver, respectively.

Under a discrete time system, the RTF can be represented by a z-transform:

$$
H\left(z, \mathbf{r}_{s}, \mathbf{r}_{o}\right)=\sum_{i=1}^{P}\left(\frac{A_{i}\left(\mathbf{r}_{s}, \mathbf{r}_{o}\right)}{1-p_{C} z^{-1}}+\frac{A_{i}^{*}\left(\mathbf{r}_{s}, \mathbf{r}_{o}\right)}{1-p_{C}^{*} z^{-1}}\right),
$$

where $p_{C}$ is a function of $\omega_{i}$ and $\delta_{i}$ but not of $r_{s}$ and $r_{0}$. Because $p_{\mathrm{C}}$ does not depend on $\mathbf{r}_{\mathrm{s}}$ and $\mathbf{r}_{\mathrm{o}}$, it is common to all RTFs in the room (i.e., it is a common acoustical pole). $P$ is the number of poles in the objective frequency band. Function $A_{d}\left(\mathbf{r}_{\mathrm{s}}, \mathbf{r}_{\mathrm{o}}\right)$ is a residue function and is expressed using $P\left(\mathbf{r}_{\mathrm{b}}\right)$ and $P\left(\mathbf{r}_{\mathrm{o}}\right)$ as

$$
A_{i}\left(\mathbf{r}_{s}, \mathbf{r}_{o}\right)=\frac{1}{2} C_{2} P_{i}\left(\mathbf{r}_{s}\right) P_{i}\left(\mathbf{r}_{o}\right)
$$

Because (2) uses the common acoustical poles and their 
residues, we call it the common-acoustical-pole and residue model.

The actual residue values can be calculated based on the common-acoustical-pole and zero model [1] because the common-acoustical-pole and residue model (2) corresponds to the partial fraction expansion [3] of the commonacoustical-pole and zero model:

$$
H\left(z, \mathbf{r}_{s}, \mathbf{r}_{o}\right)=\frac{C_{\mathrm{c}} z^{-Q_{1}} \prod_{i=1}^{Q_{2}}\left(1-q_{i}\left(\mathbf{r}_{\mathrm{s}}, \mathbf{r}_{\mathrm{o}}\right) z^{-1}\right)}{\prod_{i=1}^{P}\left(1-p_{\mathrm{C}} z^{-1}\right)},
$$

where $q\left(\mathbf{r}_{s}, \mathbf{r}_{q}\right)$ is zero and $C_{\mathrm{C}}$ is a constant. First, the common acoustical poles are estimated from the RTFs for different source and receiver positions as values common to the RTFs [1]. Next, the RTFs are modeled by the commonacoustical-pole and zero model (4). The actual value of the residue $A_{f}\left(\mathbf{r}_{s}, \mathbf{r}_{o}\right)$ can then be obtained by the following equation:

$$
A_{i}\left(\mathbf{r}_{\mathrm{s}}, \mathbf{r}_{\mathrm{o}}\right)=\left.\left(1-p_{\mathrm{C}} z^{-1}\right) \frac{C_{\mathrm{c}} z^{-Q_{1}} \prod_{n=1}^{Q_{2}}\left[1-q_{n}\left(\mathbf{r}_{s}, \mathbf{r}_{0}\right) z^{-1}\right]}{\prod_{n=1}^{P}\left(1-p_{C n} z^{-1}\right)}\right|_{z=p_{\mathrm{C}}}
$$

\section{PRINCIPLE OF INTERPOLATION AND EXTRAPOLATION OF RTF}

Because common acoustical pole $p_{\mathrm{C}}$ does not depend on the source and receiver positions in the proposed model, the RTF $H\left(z, \quad \mathbf{r}_{5}, \mathbf{r}_{0}\right)$ variations can be expressed by the variations in residue $A_{i}\left(\mathbf{r}_{5}, \mathbf{r}_{0}\right)$ in (2). Therefore, interpolation or extrapolation of the RTF at an arbitrary position can be reformulated as a problem of interpolating or extrapolating residue functions $A\left(\mathbf{r}_{8}, \mathbf{r}_{0}\right)$. Here, we will discuss this interpolation and extrapolation assuming a rectangular room because the eigenfunction of a rectangular room is well understood.

For a rectangular room, eigenfunction $P_{i}\left(\mathbf{r}_{\xi}\right)(\xi=s, 0)$ can be decomposed into three eigenfunctions of axes [2]:

$$
P_{i}\left(\mathbf{r}_{\xi}\right)=P_{x}\left(x_{\xi}\right) P_{y}\left(y_{\xi}\right) P_{z}\left(z_{\xi}\right), \text { where } \xi=\mathrm{s}, \mathrm{o}
$$

In addition, the eigenfunction of the $u-a x i s(u=x, y, z)$ can be expressed as

$$
P_{u}\left(u_{\xi}\right)=C_{u 1} \exp \left(-j k_{u} u_{\xi}\right)+C_{u 2} \exp \left(j k_{u} u_{\xi}\right)
$$

where $C_{u 1}$ and $C_{u 2}$ are constants, and $k_{u}$ is an eigenvalue expressed as

$$
k_{u}=\frac{n_{u} \pi}{L_{u}}+j \gamma_{u} \quad\left(n_{u}=1,2,3, \ldots\right)
$$

where $L_{u}$ is the dimension along the $u$-axis of the room and $\gamma_{u}$ corresponds to the acoustic absorption coefficient of the walls.

By determining parameters $C_{u 1}, C_{u 2}$, and $k_{u}$, we can obtain residue $A\left(\mathbf{r}_{s}, \mathbf{r}_{0}\right)$ at arbitrary positions $\left(\mathbf{r}_{s}, \mathbf{r}_{0}\right)$. The determination can be done by using a fitting method using the actual residues of several measured RTFs, for example, the least-squares method.

Let's now consider the case when the source location is fixed and the receiver moves parallel to one axis. For this case, the change in the residue depends only on the eigenfunction of $P_{u i}(u)$ :

$$
A_{i}(u)=C_{3} P_{u i}(u)=C_{u i} \exp \left(-j k_{u i} u\right)+C_{u i} \exp \left(j k_{u i} u\right),
$$

where $u$ represents a receiver position along the u-axis. Thus, the residue variation can be expressed by an explicit function while the zeros of the conventional commonacoustical-pole and zero model cannot.

Estimating the parameters in (9) is not easy because eigenvalue $k_{u}$ is a complex value. Therefore, in this paper we approximate residue function $A_{i}(u)$ as follows.

\section{A. Cosine function approximation of residue function}

If the acoustic absorption coefficient of the walls is small, so that eigenvalue $k_{u}$ can be treated as a real number, the residue function can be approximated as a simple cosine function:

$$
\bar{A}_{i}(u)=B_{1} \cos \left(k_{u} u+\phi\right)+B_{2} .
$$

In this cosine function approximation, two complex parameters $\left(B_{1}, B_{2}\right)$ and two real parameters $\left(k_{u}, \phi\right)$ are determined.

\section{B. AR model approximation of residue function}

A cosine wave form whose amplitude decreases or increases with time can be predicted by using a second-order $A R$ model[4]. Therefore, residue function $A(u)$ can be approximated using the second-order AR model:

$$
\hat{A}_{i}(u)=D_{1} \hat{A}_{i}(u-\Delta)+D_{2} \hat{A}_{i}(u-2 \Delta),
$$

where $D_{1}$ and $D_{2}$ are the AR parameters, and $\Delta$ is the microphone interval. In the AR model approximation, the AR parameters $\left(D_{1}\right.$ and $\left.D_{2}\right)$ and the initial conditions have to be determined using the actual residues of the RTFs observed 
at equal-interval receiver positions. This $A R$ model approximation does not disregard the amplitude variation as does the cosine function approximation.

\section{INTERPOLATION AND EXTRAPOLATION SIMULATION}

We used computer simulation to compare the performance of our methods for interpolating and extrapolating RTFs at arbitrary positions with conventional methods. Conventional interpolation estimates an RTF by linearly averaging two known RTFs. There is no specific conventional extrapolation method, but one way is to simply use the nearest known RTF as the estimated RTF.

We assumed a small rectangular room $(2.3 \times 1.3 \times 1.1 \mathrm{~m})$, about as large as the interior of a car. Room impulse responses were created using the image method [5], assuming the room had a wall-reflection coefficient of 0.93 . The sampling frequency was $800 \mathrm{~Hz}$, and the frequency range was $50-320 \mathrm{~Hz}$. The order of the poles was set to 60 . Figure 1 shows the arrangement of the source and receivers. The receiver positions were set at 16 points parallel to the $x$ axis at intervals of $10 \mathrm{~cm}$.

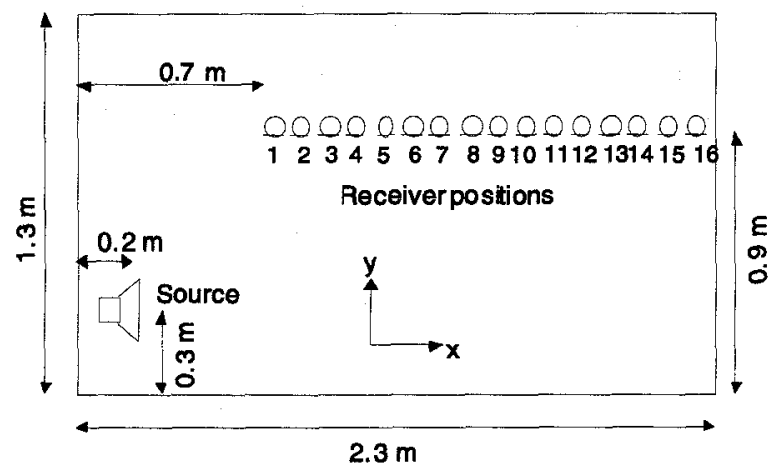

Fig. 1. Arrangement of source and receivers.

\subsection{Interpolation result}

The RTF at receiver position 7 was interpolated by using the cosine function approximation and the RTFs at receiver positions $1,2,3,11,12,13$, and 14. These RTFs were common-acoustical-pole and zero one modeled by the method in reference [1]. The common acoustical poles were calculated from these seven known RTFs. The residue values $A_{(}\left(u_{j}\right)(i=0,1, \ldots, 59 ; j=1,2,3,11,12,13$, and 14) were calculated using the partial fraction expansion in (5), and the parameters in the approximated resicue function $\hat{A}_{i}(u)$ in

(10) were estimated using the minimum mean-square-error method.

Figure 2 shows examples of the estimated residue function $\hat{A}_{i}(u)$ (dashed line) corresponding to pole frequencies of (a)

$221 \mathrm{~Hz}$ and (b) $270 \mathrm{~Hz}$. The solid line indicates the actual residues $A_{i}(u)$ calculated from the actual RTFs. The symbols $\Delta$ and $\diamond$ indicate the real and imaginary residues, respectively, used for the estimation. The estimated residue function agrees closely with the actual residues.

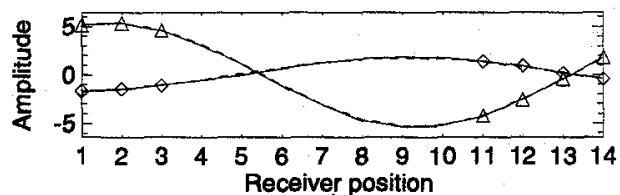

(a)

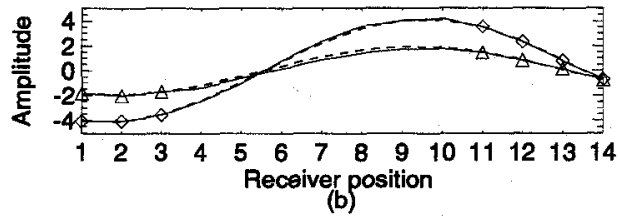

Fig. 2. Actual residues (solid line) and estimated residues (dashed line) for pole frequencies of (a) $221 \mathrm{~Hz}$ and (b) 270 $\mathrm{Hz}$.

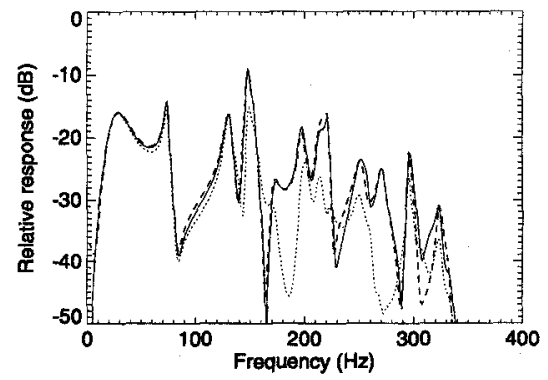

Fig. 3 Frequency responses of actual RTF (solid line) and RTFs interpolated by the proposed method (dashed line) and by conventional linear interpolation (dotted line).

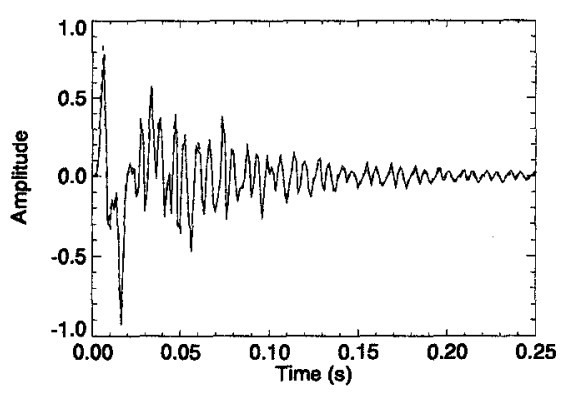

Fig. 4. Impulse responses of actual RTF (solid line) and RTF interpolated using the proposed method (dashed line).

Figure 3 shows the frequency responses of the actual RTF (solid line) and the RTFs interpolated by the proposed method (dashed line) and by conventional linear interpolation (dotted line). The RTF interpolated using our proposed method was almost equal to the actual RTF (error of $-18 \mathrm{~dB}$ ). The linearly interpolated RTF, the average of the RTFs at positions 3 and 11, had large errors at about $190 \mathrm{~Hz}$ and $270 \mathrm{~Hz}$. 
As shown in Fig. 4, the RTF interpolated by the proposed method also agreed closely with the actual RTF in the time domain.

\subsection{Extrapolation result}

We extrapolated the RTFs at receiver positions 8 to 16 by using the RTFs at positions 1 to 7 shown in Fig. 1. The two approximated residue functions (cosine function (10) and AR. model function (11)) were used to extrapolation the RTFs.

Figure 5 shows an example of the frequency response of the actual RTF (solid line) and the extrapolated RTF (dashed line) at position 11 ( $40 \mathrm{~cm}$ from receiver position 7) when the residues were extrapolated using the $A R$ model approximation. The amplitude was well estimated at each peak position.

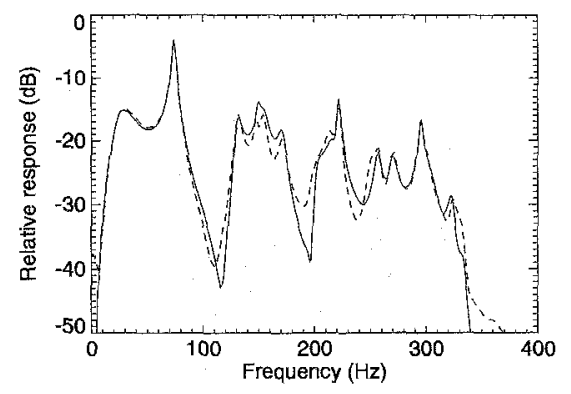

Fig. 5 Frequency responses of actual RTF (solid line) and of extrapolated RTF (dashed line) at receiver position 11 .

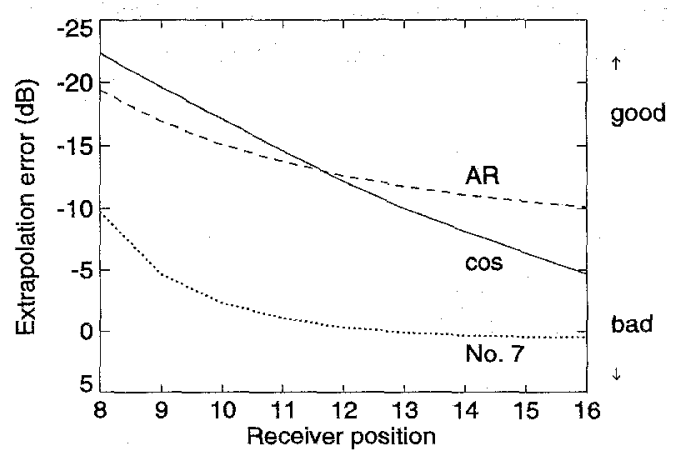

Fig. 6. Frror between the actual RTF and extrapolated RTF for each receiver position. The extrapolations were done by approximating the residue function as a cosine function or as an AR model function, or by using the values of the RTF at receiver position 7 .

We defined extrapolation error $E_{\mathrm{ex}}$ as follows:

$$
E_{e x}=\log _{10} \frac{\sum_{n=0}^{\sum_{n}}(h(n)-\hat{h}(n))^{2}}{\sum_{n=0}^{L} h(n)^{2}}(d B)
$$

where $h(n)$ is the impulse response of the actual RTF, and $\bar{h}(n)$ is the impulse response of the extrapolated RTF.

Figure 6 shows the extrapolation errors for the cosine function approximation and the AR model approximation at each receiver position. The error when the $R T F$ at receiver position 7 was used as the extrapolated RIF for all receiver positions is shown for the reference. These results show that both proposed extrapolation methods perform well. For example, the error for both methods was about $-13 \mathrm{~dB}$ at receiver position $12(50 \mathrm{~cm}$ from receiver position 7$)$, while the error using the RTF at position 7 was $0 \mathrm{~dB}$.

\section{CONCLUSION}

We have proposed a new method for modeling a room transfer function that uses the common acoustical poles and their residues. The common acoustical poles correspond to the resonance frequencies (eigenvalues), and their residues correspond to the eigenfunctions in the room.

We have also proposed methods for interpolating and extrapolating RTFs by using known RTFs based on our proposed model. Simulation of these interpolation and extrapolation methods for a rectangular room showed that proposed interpolation performance was much better than conventional linear interpolation and that the proposed extrapolation method achieved higher accuracy than using the nearest known RTF.

\section{ACKNOWLEDGMENTS}

We are grateful to Dr. N. Kitawaki, Mr. J. Kojima and Dr. S. Makino for their supports and suggestions.

\section{References}

[1] Y. Haneda, S. Makino, and Y. Kaneda, "Common acoustical pole and zero modeling of room transfer functions, "IEEE Trans. Speech Audio Process., vol. 2, pp. 320-328, April 1994.

[2] H. Kuttruf, Room Acoustics. London: Elsevier Applied Science, 1991.

[3] A. V. Oppenheim and R. W. Schafer, Discrete-time signal processing. Englewood Cliffs, NI: PrenticeHall, 1989.

[4] S. Haykin, Adaptive. Filter Theory. 2nd ed. Englewood Cliffs, NJ: Prentice-Hall, 1991.

[5] J. B. Allen and D. A. Berkly, "Image method for efficiently simulating small-room acoustics," J. Acoust. Soc. Am. 65, pp. 943-950, 1979. 